\title{
High-Speed Optical Microscopy of Spin-Like Reactions in Exothermic Nanolaminates
}

\author{
J. P. McDonald, E. D. Jones Jr., V. Carter Hodges, D. P. Adams
}

Sandia National Laboratories, Albuquerque, NM, 87185-0959

Exothermic nanolaminates are a class of energetic materials comprised of alternating layers of two or more materials which produce an exothermic reaction when they are encouraged to mix [1]. The constituent materials are grown in distinct layers, such that the exothermic energy is stored until a stimulus provides for sufficient mixing of these layers and ignition of a reaction. A schematic of an exothermic nanolaminate foil is presented in FIG. 1. In this work, the details of reaction dynamics in exothermic nanolaminates are explored at the single micrometer length scale with high speed digital photography ( $10^{5}$ frames per second), revealing the presence of spin-like reactions [2].

Initially observed during combustion of a titanium cylinder in a nitrogen gas atmosphere [3], spin reactions are thought to result from the competition between heat conduction out of the reaction volume, and heat production within the reaction volume from the exothermic chemical reaction [4]. For planar exothermic nanolaminates, spin-like reactions were initially discovered in $\mathrm{Co} / \mathrm{Al}$ foils, where transverse reaction bands were observed to propagate orthogonal to the net propagation direction [2]. Spin-like reactions have emerged as a common mode of reaction propagation, showing up in other systems including $\mathrm{Ni} / \mathrm{Ti}, \mathrm{Ni} / \mathrm{Al}$, and $\mathrm{Sc} / \mathrm{Cu}$ (an example is shown in FIG. 2). In contrast to steady reaction propagation, it was discovered that the spin-like mode of reaction propagation resulted in a periodic surface morphology present long after the reaction was complete (FIG. 3). Further, it was noted that the reaction speed in the transverse reaction bands greatly exceeded that of the net reaction propagation (FIG. 4).

The influence of exothermic foil properties on the spin-like reactions is revealed in FIG. 4, where it is apparent that both the net and transverse reaction propagation speeds decrease with increasing bilayer thickness (bilayer thickness defined in FIG. 1). This general trend was attributed to the effects of a mass diffusion limited reaction, in which atoms from the constituent layers must travel greater distance to meet and react as the bilayer thickness is increased. Recently, the combined influence of spin reactions and oxidation/combustion reactions in exothermic nanolaminates has been investigated. In Ni/Ti multilayers, for example, a reaction between the two metallic species is also accompanied by a reaction between $\mathrm{Ti}$ and $\mathrm{O}_{2}$ [5]. The oxidation reaction has the effect of increasing the rate of generation of transverse reaction bands, which has the secondary effect of increasing the net velocity of reactions performed in air.

Observation of reaction propagation in planar exothermic nanolaminates using high-speed optical microscopy revealed the presence of spin-like reactions, an unsteady mode of reaction propagation characterized by transverse reaction bands propagating orthogonal to the overall reaction direction. The characteristics of spin reactions were found to be dependent on foil design and reaction environment [6]. 


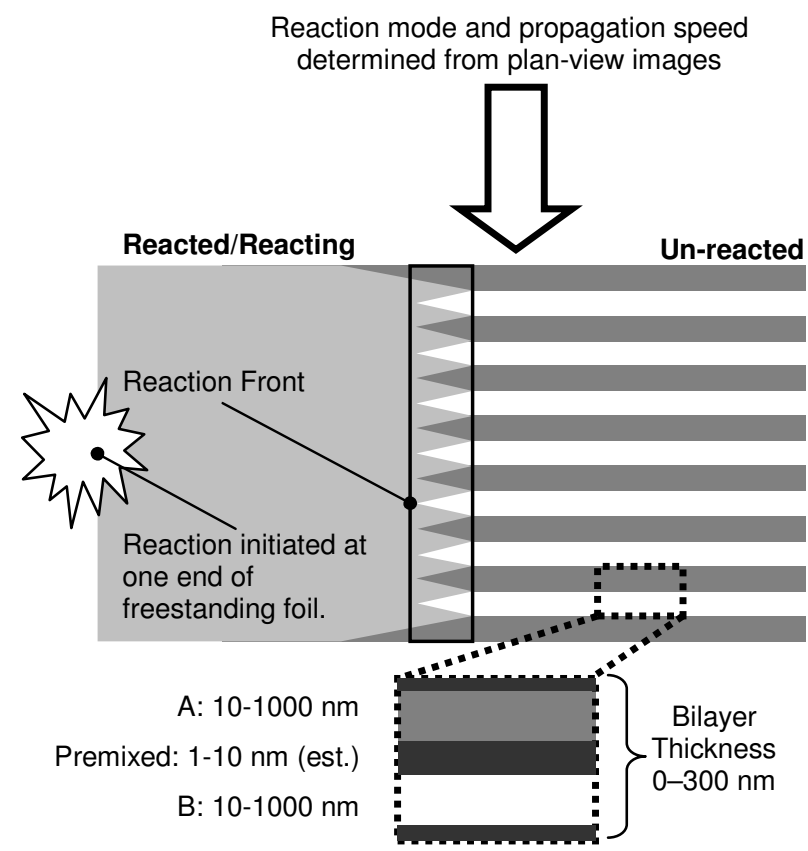

FIG 1. Schematic cross section of an exothermic nanolaminates foil. High speed camera observations of reaction dynamics are made in plan-view of the top foil surface.



FIG 3. Scanning electron micrograph of the surface of a reacted $\mathrm{Co} / \mathrm{Al}$ exothermic nanolaminate with a bilayer thickness of $250 \mathrm{~nm}$ and a total thickness of $7.5 \mu \mathrm{m}$.

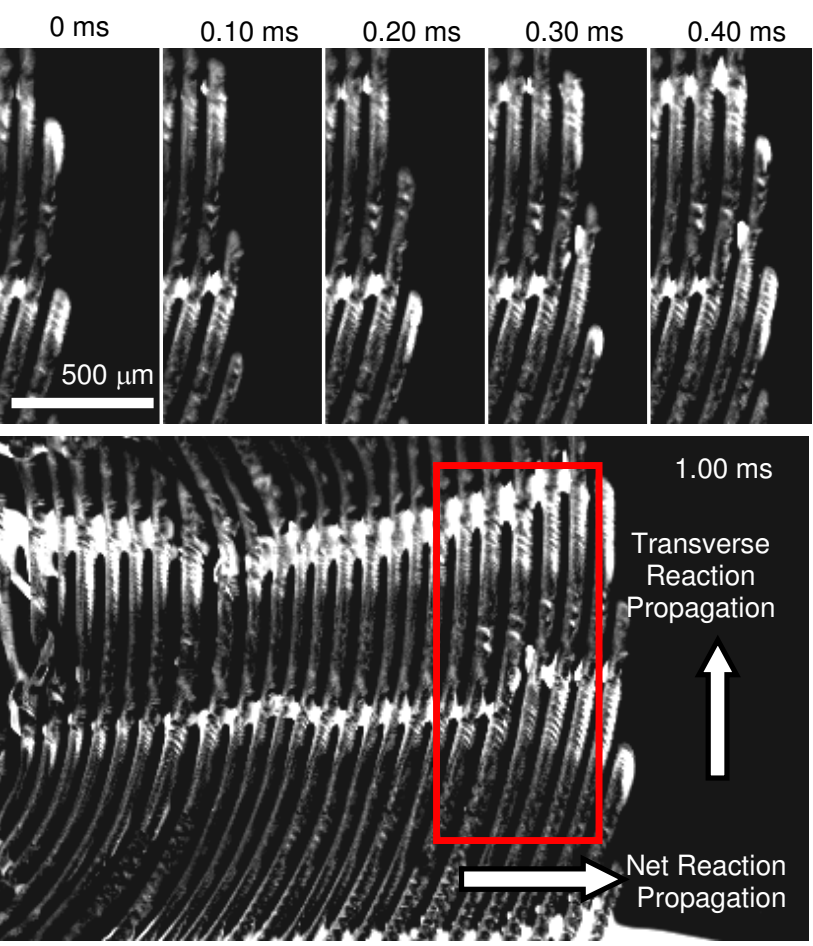

FIG 2. Still frame captures from a high speed movie of an exothermic reaction in a $\mathrm{Sc} / \mathrm{Cu}$ foil with $41.2 \mathrm{~nm}$ bilayer thickness. The time stamp in/above each image is relative to the first image in the sequence, while the scale bar in the upper left image applies to all images. Note that external illumination was used and the contrast apparent in the images is strictly reflected light.

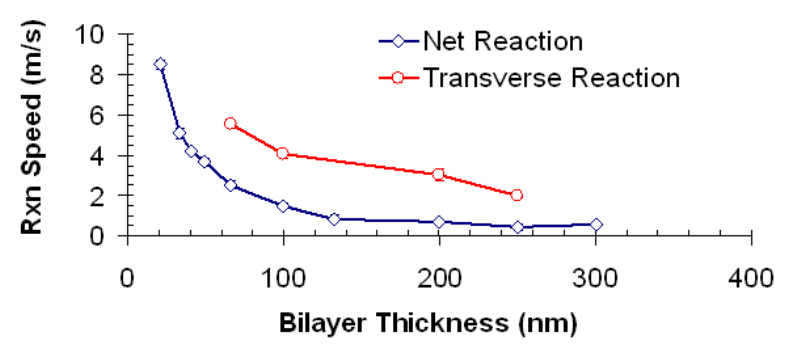

FIG 4. Net and transverse reaction speeds for $\mathrm{Co} / \mathrm{Al}$ exothermic nanolaminates as a function of bilayer thickness. All foils had a total thickness of $7.5 \mu \mathrm{m}$

\section{References:}

1. T. W. Barbee, et al, US Patent Number 5538795-A (1996).

2. J. P. McDonald, et al, Applied Physics Letters 94, 3 (2009).

3. A. K. Filonenko, et al, Combustion Explosion and Shock Waves 11, 301-308 (1975).

4. S. Gennari, et al, Journal of Physical Chemistry B 110, 7144-7152 (2006).

5. D. P. Adams, et al, Journal of Applied Physics 106, 8 (2009).

6. The authors would like to acknowledge the assistance of Kathryn A. Chinn in data collection. This work was funded by a Sandia Laboratory Directed Research \& Development program. Sandia is a multiprogram laboratory operated by Sandia Corporation, a Lockheed Martin Company, for the United States Department of Energy's National Nuclear Security Administration under contract DE-AC04-94AL85000. 